\section{Role of ventricular tachycardia ablation in arrhythmogenic right ventricular cardiomyopathy}

\author{
Alberto Cipriani, ${ }^{1}$ Riccardo Bariani, ${ }^{1}$ \\ Manuel De Lazzari, ${ }^{1}$ Federico Migliore, ${ }^{1}$ \\ Carlo Angheben, ${ }^{2}$ Maurizio Del Greco, ${ }^{2}$ \\ Domenico Corrado, ${ }^{1}$ Alessandro Zorzi ${ }^{1}$ \\ ${ }^{1}$ Department of Cardiac, Thoracic and \\ Vascular Sciences, University of Padova; \\ ${ }^{2}$ Division of Cardiology, Santa Maria del \\ Carmine Hospital, Rovereto (TN), Italy
}

\begin{abstract}
Arrhythmogenic right ventricular cardiomyopathy (ARVC) is characterized by progressive fibro-fatty replacement of the myocardium that represents the substrate for recurrent sustained ventricular tachycardia (VT). These arrhythmias characterize the clinical course of a sizeable proportion of patients and have significant implications for their quality of life and long-term prognosis. Antiarrhythmic drugs are often poorly tolerated and usually provide incomplete control of arrhythmia relapses. Catheter ablation is a potentially effective strategy to treat frequent VT episodes and ICD shocks in ARVC patients. The aims of this review are to discuss the electrophysiological and electroanatomic substrates of ventricular tachycardia in patients with ARVC and to analyze the role of catheter ablation in their management with particular reference to selection of patients, technical issues, potential complications and outcomes.
\end{abstract}

\section{Introduction}

Arrhythmogenic right ventricular cardiomyopathy (ARVC) is a rare inherited heart muscle disease characterized by progressive fibro-fatty replacement of the myocardium that represents the substrate for ventricular arrhythmias and sudden cardiac death (SCD), mostly in young individuals and athletes. ${ }^{1-4}$ It is a genetically determined cardiomyopathy, often caused by mutations in genes encoding for desmosomal proteins, which are cellular structures specialized for cell-to-cell adhesion. ${ }^{5}$ Desmosomal defects are responsible for impaired cell connections that lead over time to myocyte death, with subsequent repair by fibrofatty replacement. This pathophysiological process typically spreads from the epicardium to the endocardium, and predominantly affects the right ventricle $(\mathrm{RV})$, resulting in wall thin- ning and aneurysmal dilatation, typically located at the angles of the triangle of dysplasia, i.e. the apex, inflow and outflow tract of the RV. In the classical form of ARVC, the left ventricle (LV) is affected to a lesser extent than the RV; however, disease variants characterized by equal or greater involvement of the LV are also observed. ${ }^{6-8}$

The natural history of ARVC is characterized by a spectrum of ventricular arrhythmias ranging from premature ventricular complexes to sustained ventricular tachycardia (VT) or ventricular fibrillation (VF). ${ }^{3,9}$ The pathogenesis of ventricular arrhythmias relies on the disease phase; in younger, often previously asymptomatic patients, SCD is mainly due to VF, in the context of active phases of acute myocyte death and reactive inflammation. ${ }^{10}$ By contrast, older patients with a long-lasting disease more often experience scar-related hemodynamically stable VT, due to a reentry mechanism around a myocardial scar. ${ }^{11}$

The overall incidence of cardiac arrest attributable to VF varies among different series, ranging from $0.08 \%$ per year, ${ }^{12}$ to $3.6 \%$ per year. ${ }^{13}$ Prevention of SCD is the mainstream of ARVC therapy, and implantable cardioverter-defibrillator (ICD) therapy represents the only effective choice to achieve this aim. Many observational studies and registries of large population of ARVC patients have established the safety and efficacy of ICD therapy in interrupting lethal tachyarrhythmias and improving the survival of selected high risk ARVC patients. ${ }^{14-16}$

Another important objective of clinical management of ARVC is the improvement of symptoms and quality of life by reducing/abolishing palpitations, VT recurrences, or ICD discharges. Currently available treatment options are the pharmacological therapy and catheter ablation. While the efficacy of antiarrhythmic drugs is controversial and over the last decades no new molecules have been introduced, catheter ablation has evolved from being a poorly available and often unsuccessful treatment, to an effective therapy for most ARVC patients with recurrent sustained ventricular arrhythmias. ${ }^{17}$

The aim of this review is to provide insights into the electrophysiological and electroanatomic substrates of VT in patients with ARVC and to analyze the role of catheter ablation in their management.

\section{Indications and patient selection}

Management of ARVC patients with recurrent $\mathrm{VT}$ and frequent ICD therapy can
Correspondence: Domenico Corrado, Department of Cardiac, Thoracic and Vascular Sciences, University of Padova, via Giustiniani 2, 35128 Padova, Italy.

Tel.: +39.049.8212458 - Fax: +39.049.8761764.

E-mail: domenico.corrado@unipd.it

Key words: Endocardial voltage mapping; electrophysiology; ventricular tachycardia; sudden death.

Received for publication: 5 July 2017 .

Revision received: 4 October 2017.

Accepted for publication: 31 October 2017.

This work is licensed under a Creative Commons Attribution NonCommercial 4.0 License (CC BY-NC 4.0).

(C) Copyright A. Cipriani et al., 2017

Licensee PAGEPress, Italy

Cardiogenetics 2017; 7:6882

doi:10.4081/cardiogenetics.2017.6882

be challenging, but has significant implications for their quality of life and long-term prognosis. Antiarrhythmic drugs are often poorly tolerated and usually provide incomplete VT control. ${ }^{17}$ Catheter ablation of scar-related VT has significantly improved VT recurrence-free survival, particularly since epicardial mapping and ablation have been implemented, in addition to the more common endocardial approach. ${ }^{18}$ According to the recent International Task Force consensus statement, ${ }^{17}$ catheter ablation is considered a potentially effective strategy to treat frequent VT episodes and ICD shocks in ARVC patients. It is therefore recommended in patients with incessant VT or frequent appropriate ICD interventions on VT, despite maximal pharmacological therapy, and may be considered in patients who do not desire or cannot tolerate antiarrhythmic drugs. However, catheter ablation has not demonstrated to prevent SCD and should not be considered an alternative to ICD implantation in ARVC patients with a history of sustained VT, with the possible exception of selected cases with a drug refractory, hemodynamically stable, single morphology VT. ${ }^{17}$

\section{Electroanatomic mapping in ARVC: understanding the VT substrate}

Over the last twenty years, advances in electrophysiologic knowledge and techniques enabled a more detailed understanding of the complex arrhythmic substrate in patients with ARVC and VT. Among the tools available for direct imaging of ventricular myocardial lesions, bipolar threedimensional electroanatomic voltage mapping (3D-EVM) accurately identifies the 
presence, location, and extent of the pathological substrate of ARVC by detection of low-voltage regions, that reflect the electric consequences of transmural myocyte loss and fibrofatty replacement, observed at histopathological examinations (Figure 1). ${ }^{19}$ A good concordance between the presence and location of RV low-voltage areas identified by 3D-EVM and akinetic/dyskinetic regions detected by echocardiography or angiography was demonstrated. ${ }^{20}$ Moreover, 3D-EVM is significantly more sensitive than cardiac magnetic resonance to identify RV scar lesions..$^{20,21}$ As abnormal bipolar EVM is specific for ARVC, it can provide additional value for differential diagnosis between ARVC and other RV disease. $^{22}$

Abnormal RV endocardial regions (socalled electroanatomic scars) are defined by areas $\geq 1 \mathrm{~cm}^{2}$ with low bipolar voltages $(<1.5 \mathrm{mV})$, and fractionated potentials (i.e. $>3$ deflections, amplitude $\leq 1.5 \mathrm{mV}$, duration $>70 \mathrm{~ms}) .{ }^{23}$ Low voltage regions in patients with ARVC distinctively involve the RV free wall and particularly the anterolateral, infundibular, and inferobasal (sub-tricuspidal) region, resembling the distribution of fibrofatty scars observed in pathologic specimens. ${ }^{24}$ Low-voltage abnormalities can also be found on the apex, but typically spares the RV septum. ${ }^{22}$ By using mapping catheters with multiple poles and very small inter-electrode distance (e.g. Orion catheter $^{\mathrm{TM}}$, Boston Scientific or PentaRay $^{\mathrm{TM}}$, Biosense Webster) it is possible to create a voltage map with a very high density of points. This may allow to record electrical activities within areas of very low voltage (dense scar), revealing surviving myocardial bundles that may represent the substrate for re-entrant circuits within the scar that may otherwise be overlooked. ${ }^{25}$ Besides its diagnostic value, bipolar 3DEVM also carries prognostic significance, as one study demonstrated that the presence and extent of bipolar low voltage areas is an independent predictor of arrhythmic events, over and above classical risk factors like clinical history, arrhythmic background and ventricular dysfunction, ${ }^{19}$ and another study that the fragmented electrograms are significantly associated with arrhythmic events during follow-up. ${ }^{26}$

Endocardial bipolar EVM can fail to detect low-voltage areas in about $25-30 \%$ of ARVC patients with VT, ${ }^{19,27}$ and endocardial ablation approach provides only a modest freedom from VT recurrence. ${ }^{27}$ This is related to the pathogenesis of the disease: as proved by autopsy and pathology studies, the wavefront of fibrofatty myocardial replacement progresses from the epicardium to the endocardium, so that the scar tis- sue can either involve the whole thickness of ventricular wall or be confined to epicardial/midmural layers, sparing the endocardial regions. ${ }^{28}$ Epicardial substrate in ARVC can be better identified by epicardial voltage mapping, which is particularly useful to guide catheter ablation in patients that have failed the endocardial approach. Abnormal epicardial bipolar low-voltage areas (amplitude $<1.0 \mathrm{mV}$ ) are normally larger than the corresponding endocardial low-voltage areas, with extensive networks of late activation and fractioned potentials. ${ }^{18}$ Epicardial fat or major coronary vessel can be misclassified as abnormal, since they show low signal amplitude. To limit the possibility to misinterpret these structures as low-voltage areas, the contiguous abnormal regions should demonstrate not only low amplitude electrograms, but also discrete late potentials and/or broad multicomponent $(>80 \mathrm{~ms})$ or split signals. The presence of late potentials is specific for identification of the epicardial substrate of VT because they are not observed in patients without structural heart disease. ${ }^{29}$ Signs that could suggest the need for epicardial approach for VT mapping and ablation include a delayed intrinsicoid activation of the QRS complex of VT on surface ECG, ${ }^{30}$ the presence of isolated epicardial scar at cardiac magnetic resonance, and/or prior

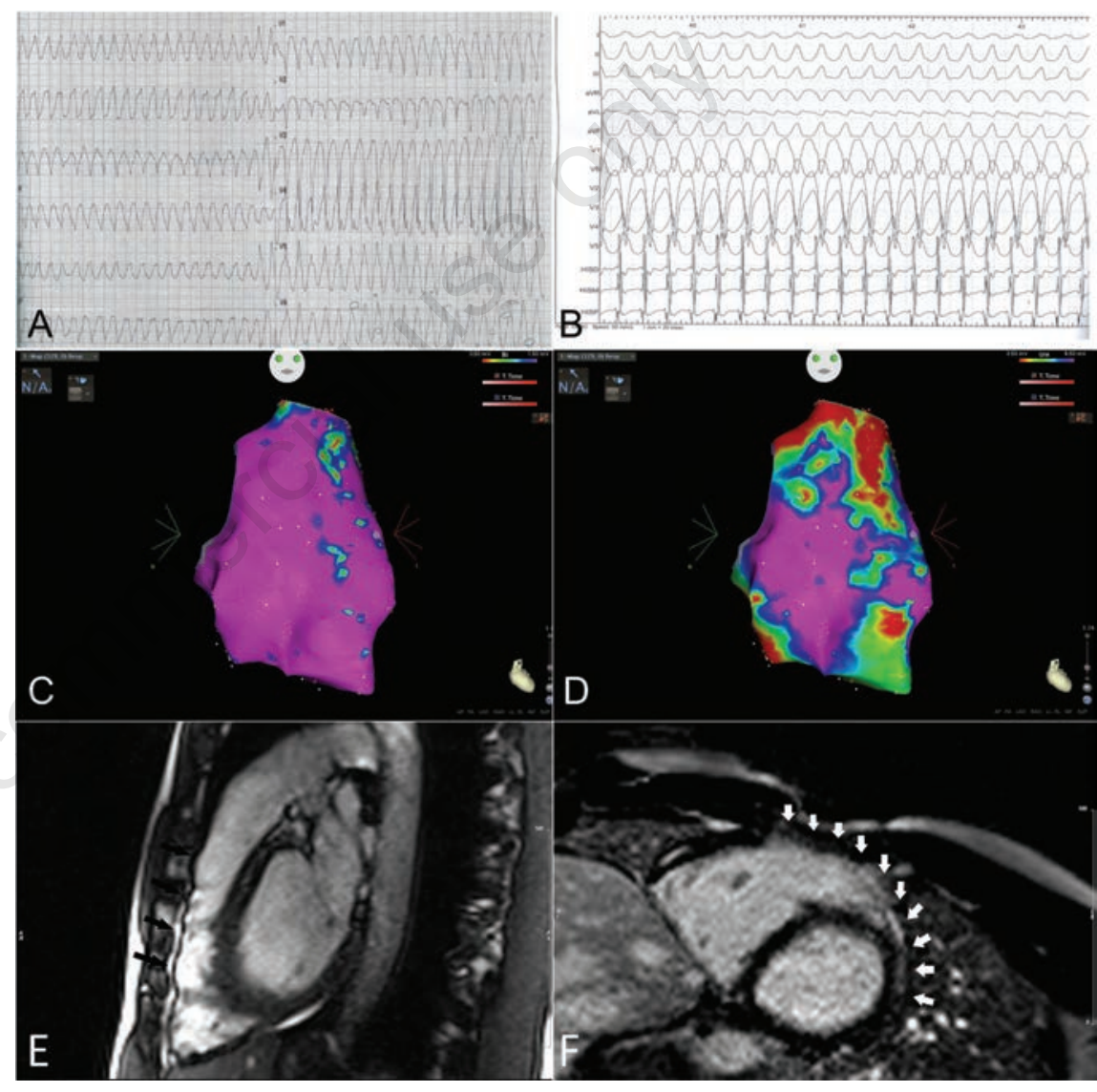

Figure 1. Representative example of a 27 year-old male who suffered hemodynamically unstable sustained ventricular tachycardia while he was running (A). The clinical tachycardia was re-induced by programmed ventricular stimulation with two extrastimuli, suggesting a re-entrant mechanism (B). The tachycardia morphology (left bundle branch block/inferior axis) suggests the origin from the right ventricular outflow tract. Anteroposterior view of right ventricular bipolar endocardial voltage mapping with the Ensite Precision system, set to show in red areas of low voltages $(<0.5 \mathrm{mV})$ and in purple areas of normal voltages $(>1.5 \mathrm{mV})(\mathrm{C})$. The exam demonstrated only limited low voltages areas, corresponding to transmural fibrofatty scar, below the pulmonary valve and in the right ventricular free wall. Instead, unipolar voltage mapping $(3.5-5.5 \mathrm{mV})$ revealed large low voltages areas, suggesting subepicardial fibrofatty replacement, involving the subtricuspid, apical and outflow tract regions (i.e. the so called triangle of dysplasia) (D). Cardiac magnetic resonance demonstrated multiple areas of systolic bulging of the right ventricular outflow tract (black arrows) (E) and late gadolinium enhancement (suggesting fibro-fatty replacement) of the anterior right ventricular wall extending to the subepicardial layer of the antero-lateral left ventricular segment (white arrows) (F). 
failed endocardial ablation. As an alternative to the invasive epicardial mapping, analysis of unipolar endocardial signals, having a larger field-of-view, can provide information about the presence of epicardial abnormality as well as identify a greater amount of low-voltage areas in those with abnormal bipolar EVM, suggesting a more extensive epicardial substrate (Figure 1D). By comparing bipolar epicardial mapping and unipolar endocardial mapping it has been demonstrated that a threshold of $<5.5$ $\mathrm{mV}$ at unipolar endocardial mapping accurately correlates with subepicardial scar regions. ${ }^{31}$

\section{Ablation technique}

The procedure of VT ablation in ARVC should be performed with almost the same general principles used in all scar-related VT ablations, but with higher chances of needing an epicardial approach. Since it has been proved that general anesthesia reduces VT inducibility, causes excessive hypotension during VT and, when combined with muscle relaxants, drops sensitivity of highoutput phrenic nerve mapping, ${ }^{32,33}$ conscious sedation is preferred over general anesthesia during the endocardial procedure. RV endocardial voltage mapping should be created in sinus rhythm with standard $0.5-1.5 \mathrm{mV}$ voltage cutoffs. ${ }^{34}$ It is crucial to provide adequate catheter contact during mapping, to confirm the observed low voltage areas. During mapping, the identification of fractionated signals and late potentials must be registered: for this purpose, modern ablation catheters with contact force sensors are particularly useful. Pace-mapping is also recommended to identify additional sites of interest. Once the RV endocardial mapping is completed, programmed ventricular stimulation can be performed to induce the VT. Electrocardiographic morphologies of induced VT should be compared to clinical arrhythmias and to pace-mapping morphologies. The standard ablation technique involves activation and entrainment VT mapping to identify the tachycardia circuit, but this technique could be problematic due to hemodynamic instability or changes in morphology caused by catheter manipulation. As an alternative, a substrate-based ablation targeting channels and delayed/fractioned potentials within low voltages areas can be performed. Radiofrequency energy is usually applied with an open irrigated tip catheter using powers up to $40 \mathrm{~W}$, for $60-90 \mathrm{sec}$, with a goal of 12 to $15 \mathrm{Ohm}$ impedance drop or $10 \%$ decrease from the baseline impedance. ${ }^{35}$ Epicardial approach is required whenever inducible VT is still present after endocardial ablation or in case of early relapse, and is generally performed with pericardial access, under general anesthesia. Mapping, electrophysiological study and ablation technique are quite similar to those described for the endocardium. The induced VTs are usually less hemodynamically tolerated so that an ablation targeting the substrate (i.e. abolishing area of low voltages and complex/fractionated potentials) is usually performed. Coronary angiography should be performed prior to epicardial ablation, to maintain a safe distance of the ablation site from main epicardial coronary vessels. ${ }^{36}$ Potential complications of epicardial ablation are cardiac tamponade, damage to coronary arteries and phrenic nerves, RV perforation, pulmonary embolism and liver laceration. ${ }^{35}$ Careful attention to blood pressure and constant monitoring of catheter position and pericardial effusion, are crucial.

\section{Outcomes}

Table 1 summarizes the main results of VT ablation in various series reported in the literature. Overall, acute success was achieved in $60-80 \%$ of patients with a rate of major complications $<5 \%$, whereas the recurrence rates during long-term follow-up of 3-5 years after a single ablation are very high, even with a combined subendocardial/subepicardial approach.

The use of a combined endocardial/epicardial approach has markedly improved acute success, which in some modern series reaches $100 \%$. As already mentioned, ARVC lesions progress from the epicardium to the endocardium, and the presence of subepicardial myocardial scars may partly

Table 1. Major series reporting ventricular tachycardia ablation outcome in arrhythmogenic cardiomyopathy.

\begin{tabular}{|c|c|c|c|c|c|c|c|c|}
\hline $\begin{array}{l}\text { Author } \\
\text { (year) }\end{array}$ & $\begin{array}{l}\text { Patients } \\
\text { n (men) }\end{array}$ & $\begin{array}{l}\text { Ablat } \\
\text { Electro-anatomic } \\
\text { map }\end{array}$ & $\begin{array}{l}\text { tion techn } \\
\text { Irrigated } \\
\text { tip }\end{array}$ & $\begin{array}{l}\text { Epicardial } \\
\text { map/abl }\end{array}$ & $\begin{array}{l}\text { Complete acute } \\
\text { success } \\
(\%)\end{array}$ & $\begin{array}{l}\text { Procedure-related } \\
\text { complications }\end{array}$ & $\begin{array}{l}\text { Fol } \\
\text { Mean } \\
\text { (months) }\end{array}$ & $\begin{array}{l}\text { VW-up } \\
\text { vT } \\
\text { recurrences (\%) }\end{array}$ \\
\hline Mussigbrodt 2017 & $45(30)$ & Yes & Yes & Yes & 84 & $\begin{array}{l}5 \text { (TIA, tamponade x2, } \\
\text { PE x2 } 1 \text { fatal) }\end{array}$ & 31 & $44^{*}$ \\
\hline Souissi 2016 & $49(44)$ & Yes & Yes & Yes & 3 & $\begin{array}{l}3 \text { (tamponade, femoral AV fistula, } \\
\text { intestinal perforation) }\end{array}$ & 64 & $\begin{array}{l}81 \text { at } 5 \text { years } \\
31 \text { at } 1 \text { years* }\end{array}$ \\
\hline Santangeli 2015 & $62(45)$ & Yes & Yes & Yes & 77 & $\begin{array}{l}5 \text { (PE x2, pericardial effusion, } \\
\text { RV punture, CT) }\end{array}$ & 56 & $29 *$ \\
\hline Philips 2012 & $86(45)$ & Yes & Yes & Yes & 82 & 2 (death, MI) & 88 & 85 \\
\hline Berruezo 2012 & $11(9)$ & Yes & Yes & Yes & 100 & 1 (tamponade) & 11 & 9 \\
\hline Garcia 2009 & $13(10)$ & Yes & Yes & Yes & 92 & 0 & 18 & 23 \\
\hline Nogami 2008 & $18(13)$ & Yes & No & No & 72 & 0 & 61 & 33 \\
\hline Dalal, 2007 & $24(11)$ & Yes & No & No & 77 & 1 (death) & 32 & 85 \\
\hline Satomi 2006 & $17(13)$ & Yes & No & No & 88 & 0 & 26 & 24 \\
\hline Verma 2005 & $22(15)$ & Yes & Yes & No & 82 & 1 (tamponade) & 37 & 36 \\
\hline Miljoen 2005 & $11(8)$ & Yes & No & No & 73 & 0 & 20 & 45 \\
\hline Marchlinski 2004 & $19(18)$ & Yes & Yes & No & 74 & 0 & 27 & 11 \\
\hline Reithmann 2003 & $5(3)$ & Yes & No & No & 80 & 0 & 7 & 20 \\
\hline Ellison 1998 & $5(4)$ & No & No & No & 42 & 0 & 17 & 0 \\
\hline
\end{tabular}

*The end-point was ventricular tachycardia-free after the last ablation. AV, artero-venous; CT, constrictive pericarditis; MI, myocardial infarction; PE, pulmonary embolism; TIA, transient ischemic attack; RV, right ventricular; VT, ventricular tachycardia. Modified with permission from Corrado D, et al. Circulation 2015;132:441-53. 
explain the failure of conventional endocardial catheter ablation. Garcia et al. ${ }^{18}$ first reported the feasibility and efficacy of epicardial ablation in ARVC patients after previously failed endocardial VT mapping/ablation procedures. In these patients, the extent of electroanatomical scar area at voltage mapping was larger on the epicardial side of the RV wall than on the endocardium. Complete success was achieved in $85 \%$ of cases (partial success in $92 \%$ ) and $77 \%$ of patients were free from VT during 18 months of follow-up. On the other hand, subepicardial ablation is more technically demanding than the traditional subendocardial approach and poses additional treats such as the risks correlated to the pericardial puncture (in the absence of pericardial fluid) and to the presence of epicardial coronary arteries. As such, it should be performed only in experienced centers.

Modern studies providing long term follow-up on large number of patients, ${ }^{37,38}$ reported very high recurrence rates after the first procedure despite a combined endo/epicardial approach, although the cumulative freedom from VT following procedures using 3D-EVM and/or epicardial approach was significantly greater than conventional ablation. The most likely reason is the progressive nature of the disease with modification of the arrhythmic substrate over time. At variance with post myocardial infarction scar, which remains relatively stable over time, the fibrofatty scar of ARVC evolves leading to the occurrence of multiple reentry circuits and new arrhythmogenic foci over time. However, recent studies ${ }^{38-40}$ reported that long-term VT-free survival can be achieved in the majority of ARVC patients, provided that multiple procedures are performed.

\section{Conclusions}

In patients with recurrent, symptomatic VT, catheter ablation has emerged as a valuable treatment option, especially in experienced centers and in cases when antiarrhythmic drug therapy is either ineffective or non- tolerated. However, compared to other diseases, ablation of VT in ARVC may be more challenging because of the subepicardial substrate and the evolving nature of the disease. Although acute success is today very good thanks to advancements in substrate characterization with 3DEVM, the use of irrigated-tip catheters with contact force sensors and epicardial approach, rates of recurrence during longterm follow-up remain high and multiple procedures are often needed to achieve good long-term results. Accordingly, VT ablation of ARVC remains a symptomatic therapy that should not be looked upon as an alternative to ICD therapy for prevention of sudden death.

\section{References}

1. Thiene G, Nava A, Corrado D, et al. Right ventricular cardiomyopathy and sudden death in young people. N Engl J Med 1988;319:174-6.

2. Basso C, Thiene G, Corrado D, et al. Arrhythmogenic right ventricular cardiomyopathy: Dysplasia, dystrophy, or myocarditis? Circulation 1996;94:9838.

3. Basso C, Corrado D, Marcus FI, et al. Arrhythmogenic right ventricular cardiomyopathy. Lancet 2009;373:1289300 .

4. Basso C, Bauce B, Corrado D, Thiene G. Pathophysiology of arrhythmogenic cardiomyopathy. Nat Rev Cardiol 2011;9:223-33.

5. Stokes DL. Desmosomes from a structural perspective. Curr Opin Cell Biol 2007;19:565-71

6. Yang Z, Bowles NE, Scherer SE, et al. Desmosomal dysfunction due to mutations in desmoplakin causes arrhythmogenic right ventricular dysplasia/cardiomyopathy. Circ Res 2006;99:64655.

7. Saffitz JE. Arrhythmogenic cardiomyopathy and abnormalities of cell-to-cell coupling. Heart Rhythm 2009;6:S6572.

8. Corrado D, Basso C, Thiene G, et al. Spectrum of clinicopathologic manifestations of arrhythmogenic right ventricular cardiomyopathy/dysplasia: a multicenter study. J Am Coll Cardiol 1997;30:1512-20.

9. Corrado D, Basso C, Pilichou K, Thiene G. Molecular biology and clinical management of arrhythmogenic right ventricular cardiomyopathy/dysplasia. Heart 2011;97:530-9.

10. Basso C, Corrado D, Bauce B, Thiene G. Arrhythmogenic right ventricular cardiomyopathy. Circ Arrhythm Electrophysiol 2012;5:1233-46.

11. Silvano M, Corrado D, Köbe J, et al. Risk stratification in arrhythmogenic right ventricular cardiomyopathy. Herzschr Elektrophys 2013;24:202-8.

12. Nava A, Bauce B, Basso C, et al. Clinical profile and long-term followup of 37 families with arrhythmogenic right ventricular cardiomyopathy. J Am Coll Cardiol 2000;36:2226-33.

13. Lemola K, Brunckhorst C, Helfenstein $\mathrm{U}$, et al. Predictors of adverse outcome in patients with arrhythmogenic right ventricular dysplasia/cardiomyopathy: Long term experience of a tertiary care centre. Heart 2005;91:1167-72.

14. Corrado D, Leoni L, Link MS, et al. Implantable cardioverter-defibrillator therapy for prevention of sudden death in patients with arrhythmogenic right ventricular cardiomyopathy/dysplasia. Circulation 2003;108:3084-91.

15. Wichter T, Paul M, Wollmann C, et al. Implantable cardioverter/defibrillator therapy in arrhythmogenic right ventricular cardiomyopathy: Single-center experience of long-term follow-up and complications in 60 patients. Circulation 2004;109:1503-8.

16. Corrado D, Calkins H, Link MS, et al. Prophylactic implantable defibrillator in patients with arrhythmogenic right ventricular cardiomyopathy/dysplasia and no prior ventricular fibrillation or sustained ventricular tachycardia. Circulation 2010;122:1144-52.

17. Corrado D, Wichter T, Link MS, et al. Treatment of arrhythmogenic right ventricular cardiomyopathy/dysplasia: an international task force consensus statement. Eur Heart J 2015;36:3227-35.

18. Garcia FC, Bazan V, Zado ES, et al. Epicardial substrate and outcome with epicardial ablation of ventricular tachycardia in arrhythmogenic right ventricular cardiomyopathy/dysplasia. Circulation 2009;120:366-75.

19. Migliore F, Zorzi A, Silvano M, et al. Prognostic value of endocardial voltage mapping in patients with arrhythmogenic right ventricular cardiomyopathy/dysplasia. Circ Arrhythm Electrophysiol 2013;6:167-76.

20. Marra M, Leoni L, Bauce B, et al. Imaging study of ventricular scar in arrhythmogenic right ventricular cardiomyopathy: comparison of 3D standard electroanatomical voltage mapping and contrast-enhanced cardiac magnetic resonance. Circ Arrhythm Electrophysiol 2012;5:91-100.

21. Santangeli P, Hamilton-Graig C, Russo $\mathrm{AD}$, et al. Imaging of scar in patients with ventricular arrhythmias of right ventricular origin: cardiac magnetic resonance versus electroanatomic mapping. J Cardiovasc Electrophysiol 2011;22:1359-66.

22. Marchlinski FE, Zado E, Dixit S, et al. Electroanatomic substrate and outcome of catheter ablative therapy for ventricular tachycardia in setting of right ventricular cardiomyopathy. Circulation 2004;110:2293-8.

23. Kiès $P$, Bootsma $M$, Bax $J$, et al. Arrhythmogenic right ventricular dys- 
plasia/cardiomyopathy: screening, diagnosis, and treatment. Heart Rhythm 2006;3:225-34.

24. Corrado D, Basso C, Leoni L, et al. Three-dimensional electroanatomic voltage mapping increases accuracy of diagnosing arrhythmogenic right ventricular cardiomyopathy/dysplasia. Circulation 2005;111:3042-50.

25. Tschabrunn CM, Rajoul S, Dorman NC, et al. High-resolution mapping of ventricular scar: comparison between single and multielectrode catheters. Circ Arrhythm Electrophysiol 2016;9:6.

26. Santangeli P, Dello Russo A, Pieroni M, et al. Fragmented and delayed electrograms within fibrofatty scar predict arrhythmic events in arrhythmogenic right ventricular cardiomyopathy: results from a prospective risk stratification study. Heart Rhythm 2012;9: 1200-6.

27. Philips B, te Riele, Sawant A, et al. Outcomes and ventricular tachycardia recurrence characteristics after epicardial ablation of ventricular tachycardia in arrhythmogenic right ventricular dysplasia/cardiomyopathy. Heart Rhythm 2015; 12:716-25.

28. Thiene G, Basso C, Calabrese F, et al. Pathology and pathogenesis of arrhythmogenic right ventricular cardiomyopathy. Herz 2000;25:210-5.

29. Cano O, Hutchinson M, Lin D, et al.
Electroanatomic substrate and ablation outcome for suspected epicardial ventricular tachycardia in left ventricular nonischemic cardiomyopathy. J Am Coll Cardiol 2009;54:799-808.

30. Roten L, Sacher F, Daly M, et al. Epicardial ventricular tachycardia ablation for which patients? Arrhythm Electrophysiolog Rev 2012;1:39-46.

31. Polin GM, Haqqani H, Tzou W, et al. Endocardial unipolar voltage mapping to identify epicardial substrate in arrhythmogenic right ventricular cardiomyopathy/dysplasia. Heart Rhythm 2011;8:76-83.

32. Aliot EM, Stevenson WG, AlmendralGarrote JM, et al. EHRA/HRS expert consensus on catheter ablation of ventricular arrhythmias. Heart rhythm 2009;6:886-94.

33. Ramoul K, Tafer N, Jais $P$, et al. Conscious sedation instead of general anesthesia for epicardial VT ablation. Arch Cardiovasc Dis Suppl 2012;4:789.

34. Hsia HH, Callans DJ, Marchlinski FE. Characterization of endocardial electrophysiological substrate in patients with nonischemic cardiomyopathy and monomorphic ventricular tachycardia. Circulation 2003;108:704-10.

35. Al-Ahmad A, Marchlinski FE. Ventricular tachycardia in structural heart disease, an issue of cardiac elec- trophysiology clinics, E-book. Saint Louis, MO: Elsevier; 2017.

36. Tschabrunn CM, Marchlinski FE. Ventricular tachycardia mapping and ablation in arrhythmogenic right ventricular cardiomyopathy/dysplasia: Lessons learned. World J Cardiol 2014;6:959-68.

37. Philips B, Madhavan S, James C, et al. Outcomes of catheter ablation of ventricular tachycardia in arrhythmogenic right ventricular dysplasia/cardiomyopathy. Circ Arrhythm Electrophysiol 2012;5:499-505.

38. Souissi Z, Boule S, Hermida JS, et al. Catheter ablation reduces ventricular tachycardia burden in patients with arrhythmogenic right ventricular cardiomyopathy: insights from a northwestern French multicentre registry. Europace 2016 [In press].

39. Santangeli P, Zado E, Supple G, et al. Long-term outcome with catheter ablation of ventricular tachycardia in patients with arrhythmogenic right ventricular cardiomyopathy. Circ Arrhythm Electrophysiol 2015;8:1413-21.

40. Müssigbrodt A, Efimova E, Knopp H, et al. Should all patients with arrhythmogenic right ventricular dysplasia/cardiomyopathy undergo epicardial catheter ablation? J Interv Card Electrophysiol 2017;48:193-9. 